\title{
NONLINEAR DIFFERENTIAL INEQUALITIES AND FUNCTIONS OF COMPACT SUPPORT
}

\author{
BY \\ RAY REDHEFFER( ${ }^{1}$ )
}

\begin{abstract}
This paper is concerned with strongly nonlinear (and possibly degenerate) elliptic partial differential equations in unbounded regions. To broaden the class of problems for which solutions exist, the equation and boundary conditions are expressed by use of set-valued functions; this involves no technical complications. The concept of "solution" is so formulated that existence is needed only in bounded regions. Uniform boundedness is first established, and compactness of support is then deduced by a comparison argument, similar to that in recent work of Brezis, but simpler in detail. The central problems here are not associated with the comparison argument, but with the nonlinearities. Our hypotheses are given only when Igrad $u \mid$ is small, so that the minimal surface operator (for example) is just as tractable as the Laplacian. Further nonlinearity is allowed by the use of the Bernstein-Serrin condition on the quadratic form, and by a suitably generalized version of the Meyers-Serrin concept of essential dimension. Although the boundary can have corners, we allow nonlinear boundary conditions of mixed type. Counterexamples show that certain seemingly ad hoc distinctions are in fact necessary to the truth of the theorems.
\end{abstract}

1. Historical resumé. It is well known that certain singular variational problems on $[0, \infty)$ have solutions with compact support. The history of this subject need not detain us; we mention only that it starts in 1952, or earlier, and continues active to the present time. In the most interesting cases the minimizing function is a spline function with infinitely many maxima and minima, the Euler equation is of the fourth order, and the maximum principles which underlie the more familiar methods of comparison and estimation do not apply.

The situation is entirely different when the Euler equation is of the second order. In that case many comparison methods are available, and the compactness can be deduced by exhibiting a super solution and subsolution, both of which themselves have compact support. This method was first used by Brezis [1]. It applies with equal ease to ordinary and partial differential equations, and gives powerful results with little calculation.

Received by the editors May 14, 1974.

AMS (MOS) subject classifications (1970). Primary 35 BXX, 35 B05, 35 B40, 35 B45, $35 \mathrm{JXX}, 35 \mathrm{~J} 25$.

$\left({ }^{1}\right)$ This research was supported in part under NSF Grant No. GP-33580X.

Copyright $\odot$ 1976, American Mathematical Society 
Our objective is to bring some of Brezis' work into contact with the theory of differential inequalities, and thus extend it to broad classes of nonlinear problems. Comparison with results of Brezis is given at appropriate points of the sequel.

2. Formulation of the problem. Let $\Omega$ be an unbounded region in $R^{n}$. Following the notation [3] we agree that $a$ denotes a symmetric $n$ by $n$ matrix, $b$ is an $n$ vector, $c$ a scalar, $u^{\prime}$ is the gradient, $u^{\prime \prime}$ the Hessian, $a u^{\prime \prime}$ and $b u^{\prime}$ denote the obvious contractions, pap is a quadratic form, $a x^{\prime}$ is the trace of $a,|x|=r$, and $u_{\nu}$ is the inner normal derivative in the sense of Walter.

We consider the relation

$$
a\left(x, u, u^{\prime}\right) u^{\prime \prime}-b\left(x, u, u^{\prime}\right) u^{\prime} \in C\left(x, u, u^{\prime}\right), \quad x \in \Omega .
$$

Appropriate boundary conditions of the form

$$
u_{\nu} \in K(x, u), \quad x \in \Gamma_{1},
$$

are prescribed on a bounded subset $\Gamma_{1}$ of $\partial \Omega$ and $u(x)=0$ on the rest of the boundary, $\Gamma_{0}$.

To deal with the fact that $\Omega$ is unbounded, let $|x|<R_{1}$ contain $\bar{\Gamma}_{1}$. The "solution" $u$ is expressed in terms of functions $u(x, R)$ for $R>R_{1}$ which satisfy (1) in the set

$$
\Omega(R)=\{x|x \in \Omega,| x \mid<R\}
$$

together with appropriate boundary conditions in this set. The latter consists of those inherited from the problem in $\Omega$ together with the additional condition $u(x, R)=0$ for $x \in \Omega,|x|=\mathrm{R}$. Although $u(x, R)$ need not exist outside of $\overline{\Omega(R)}$ it is assumed, for the time being, that $u(x, R)$ is $D^{2}$ in $\Omega(R)$ and continuous in the closure. Here $D^{1}$ is the class of differentiable functions, and $D^{2}$ is the class of functions with gradient in $D^{1}$. The problem of relaxing these conditions is discussed later.

We want to find $\rho>R_{1}$ such that every $u(x, R)$ satisfies

$$
u(x, R)=0 \text { for } \rho<|x|<R .
$$

In that case each $u(x, R)$ for $R>\rho$ gives rise to a solution of (1) and (2) in $\Omega$, and the problems of existence and uniqueness in $\Omega$ are reduced to the corresponding problems in $\Omega(R)$. This reduction of the problem to the case of a bounded region is a major goal of the theory.

Clearly the above situation cannot occur unless $C(x, 0,0)$ contains 0 for large $|x|$, and we suppose that this is the case. We also assume existence of the functions $u(x, R)$, at least for the one-sided inequalities which form the main topic of discussion. 
3. Differential inequalities. From now on $C\left(x, u, u^{\prime}\right)$ is an interval. Hence, if the left side of (1) is denoted by $T u$ then (1) can be written in the form

$$
c^{-}\left(x, u, u^{\prime}\right) \geqslant T u \geqslant c^{+}\left(x, u, u^{\prime}\right) .
$$

Strictly speaking, this requires that $C$ be a closed interval. However, when the functions $c$ are qualified only by suitable inequalities, as is done here, (3) can be made to convey exactly the same information as is conveyed by (1).

The left-hand relation (3) may be regarded as an inequality for $-u$ rather than $u$, so that both relations are included in the study of

$$
a\left(x, u, u^{\prime}\right) u^{\prime \prime}-b\left(x, u, u^{\prime}\right) u^{\prime} \geqslant c\left(x, u, u^{\prime}\right) .
$$

This is the main subject of discussion.

Corresponding to the one-sided inequality (4) are one-sided boundary conditions in $\Gamma_{1}$, formulated more precisely below. Likewise the condition on $\Gamma_{0}$ is replaced by a one-sided condition, $u(x) \leqslant 0, x \in \Gamma_{0}$. The function $u(x, R)$ is required to satisfy the inherited one-sided boundary conditions in $\Omega(R)$ together with $u(x, R) \leqslant 0, x \in \Omega(R),|x|=R$. The continuity conditions are as in $\S 2$.

If $F(R)$ denotes the family of all such one-sided solutions set

$$
\bar{u}(x)=\sup u(x, R) \text { for } u \in F(R), R>\max \left(|x|, R_{1}\right) .
$$

Then $\vec{u}(x)$ exists if and only if the family is bounded above at $x$, and the function

$$
u^{+}(x)=\max [\bar{u}(x), 0]
$$

has compact support if and only if there exists $\rho$ such that

$$
u \in F(R) \Rightarrow u(x, R) \leqslant 0 \text { for } \rho<|x|<R .
$$

It turns out that (4) is actually used only when $u>0$, and (3) only when $u \neq 0$. To avoid introducing two functions $c^{-}$and $c^{+}$we can define $c$ to agree with $c^{-}$when $u<0$, and to agree with $c^{+}$when $u>0$. The two-sided inequality is then

$$
u T u \geqslant u c\left(x, u, u^{\prime}\right) \quad(u \neq 0)
$$

and the one-sided inequality is the same, except that it holds only for $u>0$. Similar remarks apply to the boundary conditions.

4. Simple boundary conditions. For $M_{1}$ a positive constant let

$$
x \in \Gamma_{1} \text { and } u>M_{1} \Rightarrow u_{\nu}>0 .
$$

If (4) is two-sided, as explained above, the conditions are changed to

$$
x \in \Gamma_{1} \text { and }|u|>M_{1} \Rightarrow u u_{\nu}>0 .
$$


This corresponds to the following choice of $K(x, u)$ in (2):

$$
\begin{aligned}
& u<-M_{1} \Rightarrow K(x, u)=[-\infty, 0), \\
&-M_{1} \leqslant u \leqslant M_{1} \Rightarrow K(x, u)=[-\infty, \infty], \\
& u>M_{1} \Rightarrow K(x, u)=(0, \infty] .
\end{aligned}
$$

More general boundary conditions are discussed later.

To see the connection with other formulations, let $k_{i}(x, s)$ be functions on $\Gamma_{1} \times R^{1}$ with the properties

$$
|s|>M_{1} \Rightarrow s k_{1}(x, s)>0, \quad k_{2}(x, 0)=0, s k_{2}(x, s) \geqslant 0 \text { for all } s .
$$

Then (6) follows from

$$
k_{1}(x, u)=k_{2}\left(x, u_{v}\right), \quad x \in \Gamma_{1} .
$$

As a still more specific illustration, (6) would follow from

$$
\left|p u^{1 / 3}-q \sinh u_{\nu}\right| \leqslant M_{1}^{1 / 3}, \quad x \in \Gamma_{1},
$$

where $p$ and $q$ are any functions of $\left(x, u, u_{\nu}\right)$ satisfying $p \geqslant 1, q \geqslant 0$. Thus, (6) is perhaps more general than appears at first glance. It should be mentioned that (5) holds by convention if $u \leqslant M_{1}$ on $\Gamma_{1}$, and (6) if $|u| \leqslant M_{1}$ on $\Gamma_{1}$. The latter is assumed in [1].

5. Simple interior conditions. Let $R_{0}, M_{0}$ and $\epsilon$ be positive constants, with $R_{0}$ so large that $|x|<R_{0}$ contains $\Gamma_{1}$. We assume that these constants can be chosen so that the following conditions hold for $x \in \Omega$ :

$$
\begin{array}{r}
|x| \leqslant R_{0} \text { and } u>M_{0} \Rightarrow a(x, u, 0) \geqslant 0 \text { and } c(x, u, 0)>0, \\
|x|>R_{0}, u>0 \text { and }|p|<\epsilon \Rightarrow a(x, u, p) \geqslant 0 \text { and } c(x, u, 0)>0 .
\end{array}
$$

If (4) is two-sided, as explained above, the conditions are changed to

$$
\begin{array}{r}
|x| \leqslant R_{0} \text { and }|u|>M_{0} \Rightarrow a(x, u, 0) \geqslant 0 \text { and } u c(x, u, 0)>0, \\
|x|>R_{0}, u \neq 0 \text { and }|p|<\epsilon \Rightarrow a(x, u, p) \geqslant 0 \text { and } u c(x, u, 0)>0 .
\end{array}
$$

We do not require $a\left(x, u, u^{\prime}\right) \geqslant 0$ throughout $\Omega$, still less do we require $a\left(x, u, u^{\prime}\right)>0$; in other words, the results are not confined to elliptic solutions. A principal term such as

$$
a\left(x, u, u^{\prime}\right) u^{\prime \prime}=\left(1-u_{1}^{2}\right) u_{11}+\left(1-u_{2}^{2}\right) u_{22}
$$

or

$$
a\left(x, u, u^{\prime}\right) u^{\prime \prime}=u_{1}^{2} u_{11}+2 u_{1} u_{2} u_{12}+u_{2}^{2} u_{22}
$$

is allowed in the following theorems. 
6. Simple compactness theorems. In this section the boundary conditions (5) and the interior conditions (7) are called "basic assumptions." To describe further hypotheses, let $M=\max \left(M_{0}, M_{1}\right)$ and

$$
\Omega(M, \epsilon)=\{(x, u, p)|x \in \Omega, \epsilon| x|>1, M>u>0,0<| p \mid<\epsilon, p \| x\} .
$$

Clearly $\Omega(M, \epsilon)$ is a subset of $R^{2 n+1}$. However we use $\Omega(M, \epsilon)$ only in connection with a definite function $u=u(x, R)$ with $p=u^{\prime}(x, R)$, so that, in any given calculation, $\Omega(M, \epsilon)$ is correlated with a subset of $\Omega$. A statement such as " $x b(x, u, p) \leqslant o(1)$ in $\Omega(M, \epsilon)$ " means that, if $u$ and $p$ are restricted as above, then $x b(x, u, p) \leqslant \delta(r)$ where $\lim _{r \rightarrow \infty} \delta(r)=0$. (Recall that $|x|=r$, as was stated in $\S 2$.)

THEOREM 1. Besides the basic assumptions, suppose the following conditions hold in $\Omega(M, \epsilon)$ for some $\epsilon>0$ :

(i) $c(x, u, p) \geqslant \epsilon$,

(ii) $|a(x, u, p)| \leqslant o\left(r^{2}\right)$,

(iii) $x b(x, u, p) \leqslant o\left(r^{2}\right)$.

Then $u^{+}$has compact support.

The next theorems have simpler statements if the equation is suitably normalized, and they are presented in normalized form first. The modifications needed when the equation is not normalized are given in a supplementary remark at the end.

We assume that

$$
x a(x, u, p) x>0 \text { in } \Omega(M, \epsilon) .
$$

Since $p \| x$ in $\Omega(M, \epsilon)$ this is somewhat weaker than the Bernstein-Serrin condition $p a(x, u, p) p>0$ for all $p \neq 0$. The latter in turn is much weaker than the familiar assumption of uniform ellipticity.

Under hypothesis (9) let

$$
\begin{aligned}
c^{*}(x, u, p) & =\frac{c(x, u, p)}{x a(x, u, p) x}|x|^{2} \quad \text { in } \Omega(M, \epsilon), \\
d^{*}(x, u, p) & =\frac{x^{\prime} a(x, u, p)-x b(x, u, p)}{x a(x, u, p) x}|x|^{2} \quad \text { in } \Omega(M, \epsilon), \\
N=N(M, \epsilon) & =\inf d^{*}(x, u, p) \quad \text { in } \Omega(M, \epsilon) .
\end{aligned}
$$

The quantity $N$ is a nonlinear analog of the essential dimension introduced by Meyers and Serrin [2]. In the linear case $N$ is, of course, independent of $M$ and $\epsilon$. 
THEOREM 2. Besides the basic assumptions, suppose the following hold in $\Omega(M, \epsilon)$ for some $\epsilon>0$ :

(i) The essential dimension satisfies $N>-\infty$;

(ii) $c^{*}(x, u, p) \geqslant f(|x|)$ where $f>0, f \in C^{(0)}$, and the following hold for $N<2, N=2$, or $N>2$, respectively:

$$
\int_{1}^{\infty} r f(r) d r=\infty, \int_{1}^{\infty} r \log r f(r) d r=\infty, \int_{1}^{\infty} r^{N-1} f(r) d r=\infty .
$$

Then $u^{+}$has compact support.

THEOREM 3. Let $\alpha$ and $\beta$ be constants, not both 0 . Besides the basic assumptions, suppose the following hold in $\Omega(M, \epsilon)$ for some $\epsilon>0$ :

(i) $d^{*}(x, u, p) \geqslant 1-2 \alpha|x|$.

(ii) $c^{*}(x, u, p) \geqslant \beta^{2} u+f(|x|)$ where $f>0, f \in C^{(0)}$, and

$$
\int_{0}^{\infty} \exp \left\{\left[\left(\alpha^{2}+\beta^{2}\right)^{1 / 2}-\alpha\right] r\right\} f(r) d r=\infty .
$$

Then $u^{+}$has compact support.

If $\alpha=\beta=0$ condition (ii) changes to $\int_{0}^{\infty} r f(r) d r=\infty$. This is a special case of Theorem 2 .

THEOREM 4. Besides the basic assumptions, suppose the following hold in $\Omega(M, \epsilon)$ for some $\epsilon>0$ :

(i) The essential dimension satisfies $N \geqslant 1$. $C^{(0)}$, and

(ii) $c^{*}(x, u, p) \geqslant f(u) g(|p|)$ where $f(t)>0$ and $g(t)>0$ for $t>0, f, g \in$

$$
F(s)=\int_{0}^{s} f(t) d t, G(s)=\int_{0}^{s} \frac{t d t}{g(t)}
$$

exist.

(iii) With $F$ and $G$ as in (ii), $\int_{0}^{1} d s / G^{-1}[F(s)]<\infty$.

Then $u^{+}$has compact support.

The normalization underlying the above theorems consists in dividing the equation through by $\lambda\left(x, u, u^{\prime}\right)$ where

$$
x a(x, u, p) x=\lambda(x, u, p)|x|^{2} .
$$

If the new matrix so obtained is $a^{*}$ then

$$
x a^{*}(x, u, p) x=|x|^{2} \text { in } \Omega(M, \epsilon) .
$$

This operation can lead to bad behavior of the normalized coefficients $b^{*}, c^{*}, d^{*}$ and is impossible if $a x=0$. It requires some sort of lower bound for $x a x$, whereas one would like to get by with just an upper bound on $|a|$.

Given an upper bound on $|a(x, u, p)|$ in $\Omega(M, \epsilon)$, we can always normalize 
so that the new $a$ satisfies $x a^{*}(x, u, p) x \leqslant|x|^{2}$. (Just divide by $\max (\lambda, 1)$ where $\lambda$ is the function above.) The condition $x a(x, u, p) x>0$ is no longer needed; indeed $a=0$ is permitted in part or all of $\Omega(M, \epsilon)$.

Instead of introducing $a^{*}$ we assume that $a$ itself satisfies this rather harmless condition, and use the original coefficients, $b, c$ rather than $b^{*}, c^{*}$. Thus $d^{*}$ becomes

$$
d(x, u, p)=x^{\prime} a(x, u, p)-x b(x, u, p)
$$

and $N=\inf d(x, u, p)$ in $\Omega(M, \epsilon)$.

SUPPLEMENT. Let $x a(x, u, p) x \leqslant|x|^{2}$ hold in $\Omega(M, \epsilon)$ and let the hypotheses of Theorems 2, 3 or 4 be stated in terms of the original coefficients $c, d$ rather than the normalized coefficients $c^{*}, d^{*}$. Then:

(i) The conclusion of Theorem 2 holds if $N \geqslant 0$ or $f$ is decreasing.

(ii) The conclusion of Theorem 3 holds if $\alpha \leqslant 0$, or $f$ is decreasing, or $x a(x, u, p) x=\lambda|x|^{2}$ in $\Omega(M, \epsilon)$ with $\lambda$ constant, $0<\lambda \leqslant 1$.

(iii) The conclusion of Theorem 4 holds without further restriction.

7. Remarks on some results of Brezis. The relations considered in [1] have the form

$$
-a(x) u^{\prime \prime}+b(x) u^{\prime}+c(x) u+\beta(u) \ni f
$$

where $\beta$ is a maximal monotone graph in $R^{2}$ such that $\beta(0)$ is a closed interval $\left[\gamma^{-}, \gamma^{+}\right]$. With $v=-u$ the monotony of $\beta$ gives

$$
\begin{aligned}
& u>0 \Rightarrow a u^{\prime \prime}-b u^{\prime} \geqslant \gamma^{+}-f+c u, \\
& v>0 \Rightarrow a v^{\prime \prime}-b v^{\prime} \geqslant f-\gamma^{-}+c v
\end{aligned}
$$

and our results apply with obvious choices of $c(x, u, p)$. It should be observed that $a$ and $b$ are the same in both problems, so a one-sided inequality such as $b x \leqslant o\left(|x|^{2}\right)$ suffices both for $u$ and for $v=-u$.

Although the theorems of [1] are worded as existence-uniqueness theorems, actually [1] contains no new investigation of either existence or uniqueness. The whole thrust of the analysis is, as it is here, to prove compactness of support. When we suggest a relationship between our results and those of [1] we are referring to the compactness alone.

It should be mentioned too that the concept of solution in [1] is not the same as ours. However, the conditions are so worded that:

(i) The problem is locally solvable.

(ii) The maximum principle holds.

(iii) The local solution is unique.

Brezis says (i) and (iii) are "well known" in his case and he uses (ii) in the same 
way as it is used here. Our methods apply if (i) and (ii) hold. We do not need (iii), but mention that the functions $u(x, R)$ associated with (1) and (2) are unique if

$$
\begin{array}{lll}
U \in C(x, u, p), & V \in C(x, v, p), & u>v \Rightarrow U>V, \\
U \in K(x, u), & V \in K(x, v), & u>v \Rightarrow U>V .
\end{array}
$$

Other conditions can be given, especially if the Bernstein-Serrin condition holds.

With the qualifications suggested by the above remarks, it can be said that our Theorem 1 includes Theorem 1 of [1] and shows that the hypothesis that $a$ and $b$ are bounded could be replaced by the weaker conditions

$$
|a(x)| \leqslant o\left(|x|^{2}\right), \quad x b(x) \leqslant o\left(|x|^{2}\right) \text { as }|x| \rightarrow \infty .
$$

Besides generalizing the boundary conditions, we could add a term $P(x) Q(u)+$ $A(x) B\left(u^{\prime}\right)$ (for example) to the left side of (10), under the sole assumption that $P(x) \geqslant 0, u Q(u) \geqslant 0,|A(x)|$ is bounded, $B$ is continuous at 0 , and $B(0)=0$. We could also replace the term $c(x) u$ in $(10)$ by a nonlinear term, such as $c(x) u^{3}$.

Theorem 3 shows that the hypothesis that $|b(x)|$ is bounded in Theorem 1' of [1] could be replaced by the weaker hypothesis $x b(x) \leqslant o(|x|)$. Furthermore the conditions that

$$
\lim e^{\delta r}\left[\gamma^{+}-f(x)\right]=\infty, \quad \lim e^{\delta r}\left[\gamma^{-}-f(x)\right]=-\infty
$$

for every $\delta>0$ could be replaced by

$$
\gamma^{+}-f(x) \geqslant F(r), \quad f(x)-\gamma^{-} \geqslant G(r)
$$

where $F$ and $G$ are suitably restricted functions satisfying $F \geqslant 0, G \geqslant 0$ and

$$
\int_{0}^{\infty} e^{\delta r} F(r) d r=\infty, \int_{0}^{\infty} e^{\delta r} G(r) d r=\infty
$$

for every $\delta>0$. In the general case $F$ and $G$ have to be monotone and (14) is equivalent to

$$
\lim \sup e^{\delta r} F(r)=\infty, \quad \lim \sup e^{\delta r} G(r)=\infty
$$

for each $\delta>0$. This is only a slight improvement on (12). However, if $\alpha \leqslant 0$ in Theorem 3, or if Brezis' equation is so normalized that $x a(x) x=\lambda|x|^{2}$, $\lambda$ const, $0 \leqslant \lambda \leqslant 1$, then the monotony of $F$ and $G$ is not required, and the improvement represented by (14) is more substantial. In addition, Theorem 3 gives information about the dependence of $\delta$ on the other parameters of the problem, and it embraces nonlinear situations not contemplated in [1].

Theorem 2 shows that the hypothesis 


$$
\lim r^{n}\left[\gamma^{+}-f(x)\right]>0, \quad \lim r^{n}\left[\gamma^{-}-f(x)\right]<0
$$

in Theorem 2 of [1] can be replaced by (13) where

$$
\int_{0}^{\infty} r \log r F(r) d r=\infty \quad(n=2), \int_{0}^{\infty} r^{n-1} F(r) d r=\infty \quad(n \geqslant 3) .
$$

Although Theorem 2 in [1] is just for the Laplacian our Theorem 2 applies to the general elliptic-parabolic operator $a u^{\prime \prime}-b u^{\prime}$ and shows that the relevant parameter is the essential dimension $N$, not the dimension $n$ of the space. Also, as in the other cases, our result applies to nonlinear situations.

This application of Theorem 2 is subject to a minor adjustment described now. Since the term $c(x) u$ is missing, the hypothesis in Theorem 2 of [1] does not entail our "basic hypothesis" on $c(x, u, p)$ in $|x| \leqslant R_{0}$; it gives only $c(x, u, p)>-\gamma$ where $\gamma$ is some constant. However, if

$$
\inf _{|x| \leqslant R_{0}}\left[x^{\prime} a(x)-x b(x)\right]>0
$$

and if the Dirichlet boundary condition $|u| \leqslant M_{1}$ on $\Gamma_{1}$ is assumed, as in [1], it is easy to show that the condition $c(x, u, p)>-\gamma,|x| \leqslant R_{0}$, suffices. In [1] the expression (15) is $n$.

We do not pursue this topic because under Brezis' conditions on $a$ and $b$ one can usually get by with $c(x, u, p) \geqslant-\gamma(1+|p|),|x| \leqslant R_{0}$, and one can allow mixed boundary conditions substantially more general than those of $\$ 5$. These matters are discussed later. It will be seen that the main requirement is the Bernstein-Serrin condition mentioned above.

Theorem 4 is not compared with [1] because the latter has no analog of Theorem 4. Previous results showing closest affinity to Theorem 4 are certain conditions for nonexistence of entire solutions [3].

8. An elementary lemma. We introduce the abbreviations

$$
u=u(x, R), \quad a=a\left(x, u, u^{\prime}\right), \quad b=b\left(x, u, u^{\prime}\right), \quad c=c\left(x, u, u^{\prime}\right)
$$

and the state following differential inequality and side condition for a radial function $\mu(r)$ :

$$
\frac{x a x}{|x|}\left(\frac{\mu^{\prime}}{r}\right)^{\prime}+\left(a x^{\prime}-b x\right) \frac{\mu^{\prime}}{r}<c, \quad a \geqslant 0 .
$$

This is to hold in the subset of $\Omega(R)$ where simultaneously

$$
r_{0}<|x|<\rho, \quad|x| u^{\prime}(x, R)=x \mu^{\prime}(|x|), \quad u(x, R)>\mu(|x|),
$$

$r_{0}$ being any sufficiently large constant. We require in addition

$$
\mu\left(r_{0}\right) \geqslant M, \quad \mu(\rho)=\mu^{\prime}(\rho)=0, \quad \mu(r) \geqslant 0 \quad\left(r_{0}<r<\rho\right) .
$$


LEMMA 1. With notation as above let $u(x, R) \leqslant M$ for $|x|=r_{0}$. Then $u(x, R) \leqslant 0$ for $\rho<|x|<R$.

For proof let $\mu(r)$ be defined as 0 for $r>\rho$. Clearly $u(x, R) \leqslant \mu(r)$ on the boundary of the set $\left\{x\left|x \in \Omega(R), r_{0}<\right| x \mid<R\right\}$ and hence, by Theorem 2 of [3], the same inequality holds inside. This gives the conclusion.

9. Proof of Theorem 1. Let sup $u(x, R)=M^{*}$. If $M^{*}>M_{1}$ the boundary hypothesis shows the value $M^{*}$ is not attained in $\Gamma_{1}$ and if $M^{*}>M_{0}$ the interior hypothesis shows it is not attained in $\Omega(R)$. Hence $M^{*} \leqslant M$ or, in other words,

$$
u(x, R) \leqslant M=\max \left(M_{0}, M_{1}\right) .
$$

This result is used without further comment in the sequel.

Now let

$$
\mu(r)=M\left[2-r / r_{0}\right]^{2}, \quad r_{0}<r<2 r_{0}=\rho,
$$

taking $r_{0}$ so large that $r_{0}>R_{0}, \epsilon r_{0}>1, \epsilon r_{0}>2 M$. These inequalities give the side conditions associated with $\Omega(M, \epsilon)$ when Lemma 2 is applied, and hence the differential inequality for $\mu$ holds if

$$
\frac{x a x}{|x|^{2}} \frac{4 M}{r_{0} r}-\left(a x^{\prime}-b x\right) \frac{2 M}{r_{0} r}\left(2-\frac{r}{r_{0}}\right)<\epsilon .
$$

This is assured for large $r_{0}$ by conditions (ii) and (iii) in the theorem.

The proof leads to a number of similar theorems, of which Theorem 1 is perhaps the simplest example. In $\Omega(M, \epsilon)$ let

$$
x a x \leqslant \alpha(r) r^{2}, \quad b x \leqslant a x^{\prime}+\beta(r), \quad c \geqslant \gamma(r)
$$

where $r=|x|$ and $\alpha, \beta, \gamma$ are given continuous functions. Then if there is a suitable solution $\theta$ of the first-order linear inequality

$$
\alpha(r) \theta^{\prime}(r)+\beta(r) \theta(r)<\gamma(r), \quad \theta(\rho)=0,
$$

the compactness follows by taking $\mu^{\prime}=r \theta$. Theorem 2 , which is discussed next, corresponds to the case $\alpha=1, \beta=$ const. The supplement to Theorem 2 corresponds to $0 \leqslant \alpha \leqslant 1, \beta=$ const.

The main difference between our proof and the proof of Theorem 1 in [1] is that we separate the problems of getting an a priori bound for $u$, on the one hand, and showing compactness of support on the other. In [1] these steps are intertwined, and the method is less general, although it involves more computation. Actually, our result cannot easily be obtained by following Brezis' procedure even when the interior operator $T$ and the boundary operator are both linear. The trouble is twofold: the estimate for $M$ tends to $\infty$ as $r_{0} \rightarrow \infty$, and 
the use of a radial comparison function near $\Gamma_{1}$ does not lend itself to mixed boundary conditions.

10. Proof of Theorem 2, easy case. In this section we ignore the restrictions associated with $\Omega(M, \epsilon)$. Under the hypothesis of Theorem 2, the differential inequality of Lemma 1 is satisfied if

$$
\mu^{\prime \prime}+(N-1) \mu^{\prime} / r<f(r), \quad \mu^{\prime} \leqslant 0 .
$$

By using $2 f$ rather that $f$ we see that (19) can be solved as an equality; the strict inequality is not necessary. (The same can be demonstrated under the hypothesis that $f \geqslant 0$, but the matter is then less trivial.) Accordingly, we find the solution of the differential equation satisfying $\mu(\rho)=\mu^{\prime}(\rho)=0$. For $N \neq 2$ this is

$$
\mu(r)=\int_{r}^{\rho} \frac{t^{2-N}-r^{2-N}}{2-N} t^{N-1} f(t) d t, \quad r_{0}<r<\rho .
$$

The case $N=2$ is similar and is not considered here.

The equation

$$
\mu^{\prime}(r)=-r^{1-N} \int_{r}^{\rho} t^{N-1} f(t) d t
$$

shows that $\mu^{\prime}(r) \leqslant 0$ and that $\left(\mu^{\prime} / r\right)^{\prime} \geqslant 0$ if $N \geqslant 0$ or if $f(t)$ is decreasing. (This last is necessary for the supplementary remark.) Divergence of the integrals in Theorem 2 implies divergence of (20) as $\rho \rightarrow \infty$, and hence the condition $\mu\left(r_{0}\right)$ $=M$ can be attained.

11. Proof of Theorem 2, continued. The foregoing argument generalizes Theorem 2 of [1], as noted above. However, from the point of view of this paper it does not come to grips with the main problem, which is to ensure that $(x, u, p)$ belongs to $\Omega(M, \epsilon)$ when Lemma 1 is applied. This problem is discussed now.

LEMMA 2. Let $w$ and $f$ be locally integrable functions from $[0, \infty)$ to $[0, \infty)$ such that

$$
\lim _{t \rightarrow \infty} w(t)=\infty \text { and } \int_{0}^{\infty} w(t) f(t) d t=\infty .
$$

Then there exists a minorant $g(t), 0 \leqslant g(t) \leqslant f(t)$, such that

$$
\int_{0}^{\infty} w(t) g(t) d t=\infty \text { and } \int_{0}^{\infty} g(t) d t<\infty .
$$

Furthermore if $f$ was monotone decreasing we can choose $g$ to be monotone decreasing, if $f$ was continuous, $g$ can be continuous, and so on.

We assume that $w f$ is locally integrable since otherwise the result is obvious. Pick a sequence $\{t(n)\}$ such that $w(t) \geqslant n^{2}$ for $t \geqslant t(n)$, taking $t(0)=0$. If 


$$
\int_{t(n)}^{t(n+1)} f(t) d t \leqslant \frac{1}{(n+1)^{2}}
$$

holds for $n=0,1,2, \ldots$ use $g(t)=f(t)$. Otherwise let $n_{0}$ be the first $n$ where inequality (22) fails and multiply $f_{0}(t) \equiv f(t)$ by a constant $c_{0}$ on the interval $\left[t\left(n_{0}\right), \infty\right)$ so equality holds in (22) at $n=n_{0}$. This operation produces a new function, $f_{1}$. If (22) holds for $f_{1}$ we take $g=f_{1}$. If not, let $n_{1}$ be the first $n$ where (22) fails for $f_{1}$, multiply $f_{1}$ by a constant $c_{1}$ beyond $t\left(n_{1}\right)$ to get equality in (22) at $n=n_{1}$, and call the resulting function $f_{2}$. Continuing in this way, we get a sequence of functions $f_{0}, f_{1}, f_{2}, \ldots$ which has a limit $g$. It is easily checked that $g$ has the desired properties, or allows a suitable smooth minorant which has them.

When Lemma 2 is applied to the proof of Theorem 2 our requirements are

$$
\begin{gathered}
\int_{r_{0}}^{\rho}\left(t^{2-N}-r_{0}^{2-N}\right) t^{N-1} g(t) d t=M(2-N), \\
-\mu^{\prime}(r)=r^{1-N} \int_{r}^{\rho} t^{N-1} g(t) d t<\epsilon, \quad r_{0}<r<\rho .
\end{gathered}
$$

If $N<2$ we choose $g$ by the lemma so that $\int_{0}^{\infty} \operatorname{tg}(t) d t=\infty$ and so that

$$
\int_{0}^{\infty} t^{N-1} g(t) d t<\epsilon \text { or } \int_{0}^{\infty} g(t) d t<\epsilon
$$

for $1 \leqslant N<2$ or $N \leqslant 1$, respectively. The possibility of getting a suitable $\rho$ is easily verified. If $N>2$ we choose $g$ so that

$$
\int_{0}^{\infty} t^{N-1} g(t) d t=\infty, \int_{0}^{\infty} \operatorname{tg}(t) d t<1 .
$$

Since the curve is convex for $N \geqslant 1$ it suffices to have $\left|\mu^{\prime}\left(r_{0}\right)\right|<\epsilon$, and on the other hand the condition involving $M$ holds if

$$
r_{0}^{2-N} \int_{r_{0}}^{\rho} t^{N-1} g(t) d t \geqslant M(N-2)+1 .
$$

First we choose $r_{0}$ so large that $M(N-2)+1<r_{0} \epsilon$, and then choose $\rho$ so that (23) holds as an equality. Since the left side of (23) is $r_{0}\left|\mu^{\prime}\left(r_{0}\right)\right|$ this completes the proof.

12. Proof of Theorem 3, easy case. As in the proof of Theorem 2 above, we begin by ignoring the restrictions associated with $\Omega(M, \epsilon)$. The differential inequality of Lemma 1 holds in Theorem 3 if $\mu$ satisfies

$$
\mu^{\prime \prime}-2 \alpha \mu^{\prime}-\beta^{2} \mu=f(r), \quad \mu^{\prime} \leqslant 0, \quad \mu \geqslant 0 .
$$

Hence we consider the solution of this equation satisfying $\mu(\rho)=\mu^{\prime}(\rho)=0$; namely, we consider

$$
\mu(r)=\int_{\rho}^{r} \frac{e^{\sigma(r-t)}-e^{\tau(r-t)}}{\sigma-\tau} f(t) d t,
$$


where $\tau$ and $\sigma$ are the roots of $s^{2}-2 \alpha s-\beta^{2}=0$, with $\tau<0<\sigma$. This assumes $\beta>0$. The case $\beta=0$ is easier and is not presented here.

Divergence of the integral in (ii) of Theorem 3 implies divergence of the integral above, and hence the condition $\mu\left(r_{0}\right)>M$ can be attained with $\epsilon r_{0}>1$ and with a suitable $\rho$. The conditions $\mu \geqslant 0, \mu^{\prime} \leqslant 0$ are verified by inspection, and Theorem 3 follows.

To get the supplementary remark note that the differential equation gives $\mu^{\prime} \geqslant 0$ whenever $\alpha \leqslant 0$. If $\alpha>0$ and $f$ is decreasing use the explicit formula

$$
\mu^{\prime \prime}(r)=f(r)-\int_{r}^{\rho} \frac{\sigma^{2} e^{\sigma(r-t)}-\tau^{2} e^{\tau(r-t)}}{\sigma-\tau} f(t) d t .
$$

By the second mean-value theorem (or, more simply, by considering the worst $f)$ it is seen that $\mu^{\prime \prime}(r) \geqslant 0$ if

$$
\int_{r}^{s} \frac{\sigma^{2} e^{\sigma(r-t)}-\tau^{2} e^{\tau(r-t)}}{\sigma-\tau} d t \leqslant 1, \quad r<s<\infty .
$$

That this is so is readily verified by taking $y=t-r$ as new variable and evaluating the integral.

The only remaining case is that $x a x=\lambda|x|^{2}$ and $\alpha>0$. If $\gamma$ is a given positive number the locus $\left\{(\alpha, \beta) \mid\left(\alpha^{2}+\beta^{2}\right)^{1 / 2}-\alpha \geqslant \gamma\right\}$ is equivalent to the locus $\left\{\left(\alpha, \beta^{2}\right) \mid \beta^{2} \geqslant \gamma(\gamma+2 \alpha)\right\}$. The latter is a closed half plane, not containing the origin. Suppose, now, that $\left(\alpha, \beta^{2}\right)$ belongs to this locus, and that $0<\lambda \leqslant 1$. Then $\left(\alpha / \lambda, \beta^{2} / \lambda\right)$ also belongs to the locus. This shows that the case $0<\lambda<1$ allows a larger exponent $-\tau$ than the case $\lambda=1$ and completes the proof.

In the present context we can go further and even allow $\lambda=0$. (This possibility was already implied in the above discussion of Brezis' equation.) As $\lambda \rightarrow 0$ the limiting value of the exponent $-\gamma(\lambda)$ is $\beta^{2} / 2 \alpha$, which is larger than the exponent in Theorem 3. Hence $\int_{0}^{\infty} \exp \left\{\left(\beta^{2} / 2 \alpha\right) t\right\} f(t) d t=\infty$ and we can construct a solution of

$$
-2 \alpha \mu^{\prime}-\beta^{2} \mu \leqslant f(t), \quad \mu(\rho)=0, \quad \mu\left(r_{0}\right) \geqslant M .
$$

Replacing $f$ by a differentiable minorant that vanishes at $\rho=\rho_{i} \rightarrow \infty$ ensures $u \in C^{(2)}, \mu^{\prime}(\rho)=0$, and the desired conclusion follows.

The only reason for excluding the case $\lambda=0$ in the Supplement is that we would have to assume $f$ bounded to get into the set $\Omega(M, \epsilon)$. This matter is discussed next.

13. Proof of Theorem 3, continued. We now re-examine the proof of Theorem 3 with a view to constructing a function $\mu$ with $\left|\mu^{\prime}\right|$ small.

Since $\sigma(r-t)<\tau(r-t)$ for $r<t<\rho$ it follows that

$$
\left|\mu^{\prime}(r)\right| \leqslant \int_{r}^{\rho} e^{\tau(r-t)} f(t) d t, \quad r_{0}<r<\rho .
$$


On the other hand the condition $\mu(r) \leqslant M$ is compatible with $\mu\left(r_{0}\right) \geqslant M$ and gives

$$
\int_{r}^{\rho} e^{\tau(r-t)} f(t) d t \leqslant(\sigma-\tau) M+\int_{r}^{\rho} e^{\sigma(r-t)} f(t) d t .
$$

To get a useful result, we have to arrange matters so that $M$ is small and also so that the second integral in (27) is small. This is accomplished by combining Lemma 2 with the following lemma:

LEMma 3. Let $u(x)$ be differentiable and $\phi(r)$ continuous. For some value $r_{1}$ let the one-sided derivatives satisfy $\phi^{\prime}\left(r_{1}+\right)<\phi^{\prime}\left(r_{1}-\right)$. Then $u(x)-\phi(r)$ does not attain its maximum over the region $x \in \Omega, r_{0}<|x|<r_{2}$, at any point of the surface $|x|=r_{1}$ which is interior to $\Omega$.

We omit the trivial proof.

Besides these lemmas, we shall also need an estimate for $\left|\mu^{\prime}(r)\right|$ from $\left|\mu^{\prime}\left(r_{0}\right)\right|$, even if $\mu^{\prime \prime}<0$ at some points. This is obtained by combining (26) at $r$ with

$$
\left|\mu^{\prime}(r)\right| \geqslant \int_{r}^{\rho} \frac{|\tau| e^{\tau(r-t)}}{\sigma-\tau} f(t) d t
$$

at $r_{0}$. The two results together give

$$
|\tau|\left|\mu^{\prime}(r)\right| \leqslant(\sigma-\tau)\left|\mu^{\prime}\left(r_{0}\right)\right|, \quad r_{0}<r<\rho .
$$

Turning now to the proof of Theorem 3 , we assume $\alpha \neq 0, \beta \neq 0$, $2(\sigma-\tau) M>\epsilon$. (If one or more of these conditions fails the proof simplifies.) Choose constants $m$ and $r_{0}$ such that $2(\sigma-\tau) m=\epsilon, \epsilon r_{0}=2$, and let $y=\mu_{0}(t)$ $=\kappa_{0}-\kappa t$ be the equation of the line segment joining $\left(r_{0}, M\right)$ to $\left(r_{1}, m\right)$, where $r_{1}>r_{0}$. We make $r_{1}$ so large that the slope $-k$ of this line satisfies

$$
2|\alpha|_{\kappa}<\beta^{2} m, 2(\sigma-\tau) \kappa<\epsilon|\tau| .
$$

The first of these inequalities ensures

$$
\mu_{0}^{\prime \prime}-2 \alpha \mu_{0}^{\prime}-\beta^{2} \mu_{0} \leqslant 0 .
$$

The second inequality (29) is used later. It is important to note that both inequalities continue to hold as $m$ increases, provided $m \leqslant M$, and also that $\kappa$ decreases as $m$ increases.

Now that $r_{1}$ is fixed, use Lemma 2 to construct a continuous minorant $g$ such that

$$
f(t) \geqslant g(t) \geqslant 0, \quad \int_{0}^{\infty} e^{-\tau t} g(t) d t=\infty, \quad \int_{0}^{\infty} g(t) d t<\frac{\epsilon}{2} .
$$

If $f(t)$ was monotone, we take $g(t)$ also monotone.

Let $\mu_{1}(t)$ satisfy 


$$
\mu_{1}^{\prime \prime}-2 \alpha \mu_{1}^{\prime}-\beta^{2} \mu_{1}=g(t), \quad \mu_{1}\left(r_{1}\right)=m, \quad \mu_{1}(\rho)=\mu_{1}^{\prime}(\rho)=0
$$

for some $\rho>r_{1}$. The existence of $\mu_{1}$ follows from the divergence of the relevant integral, as in $\S 12$. The function $\mu$ defined by

$$
\mu(t)=\mu_{0}(t) \quad\left(r_{0} \leqslant t \leqslant r_{1}\right), \quad \mu(t)=\mu_{1}(t) \quad\left(r_{1} \leqslant t \leqslant \rho\right)
$$

satisfies the appropriate differential inequality and boundary conditions, except perhaps at $t=r_{1}$, and also satisfies

$$
\mu^{\prime \prime} \geqslant 0, \quad \mu^{\prime} \leqslant 0, \quad \mu \geqslant 0, \quad\left|\mu^{\prime}\right|<\epsilon \quad\left(t \neq r_{1}\right) .
$$

Here the first three conditions follow as in $\S 12$ and the last follows from (26) and (27) applied to $\left(m, r_{1}, g\right)$ instead of $\left(M, r_{0}, f\right)$.

If $\mu_{1}^{\prime}\left(r_{1}+\right) \leqslant-2 \kappa$, where $-\kappa$ is the slope of the line $\mu_{0}$ as mentioned above, then Lemma 3 shows that the function $u(x)-\mu(|x|)$ does not attain its maximum at an interior point where $|x|=r_{1}$. Hence the maximum, if positive, is attained at an interior point where $r_{0}<|x|<r_{1}$ or $r_{1}<|x|<\rho$. Either case leads to a contradiction as in $\S 12$. However, we now have $\left|\mu^{\prime}(t)\right|<\epsilon$ in the maximum point, so that the point is in $\Omega(M, \epsilon)$.

Suppose next that $\mu_{1}^{\prime}\left(r_{1}+\right)>-2 \kappa$. As we increase the value $m$, the value of $\rho$ also increases, hence $\left|\mu_{1}^{\prime}\left(r_{1}\right)\right|$ increases, and on the other hand $\kappa$ decreases. Since the functions are continuous, and the finai value of $k$ is 0 , there must be a point at which equality holds. For this value of $m$, say $m=m_{2}$, and for the corresponding $\mu_{2}$ on $\left(r_{1}, \rho_{2}\right)$, we have

$$
\left|\mu_{2}\left(r_{1}+\right)\right|=2 \kappa_{2} \leqslant \epsilon|\tau| /(\sigma-\tau)
$$

where the last equality is the second inequality (29). Applying (28) to $\left(\mu_{2}, r_{1}\right.$, $\left.\rho_{2}\right)$ instead of $\left(\mu, r_{0}, \rho\right)$ we get $\left|\mu_{2}^{\prime}(r)\right|<\epsilon, r_{1}<r<\rho_{2}$. The proof follows as in the first case.

It should perhaps be mentioned that these complications are not necessary to generalize Theorem $1^{\prime}$ of [1] as explained above. The latter follows from the argument in $\S 12$.

14. A counterexample. We discuss Theorem 3 when $\alpha>0$ and the equation is not normalized. In the linear case, which is assumed here, the basic condition is

$$
x a(x) x=\lambda(x)|x|^{2}, \quad 0 \leqslant \lambda(x) \leqslant 1 .
$$

If $\lambda$ is constant the equation behaves like the normalized equation, for which $\lambda=1$. But if $\lambda$ is variable, the proof of Theorem 3 requires that $f$ be monotone. We want to show that some condition of the sort is necessary; in other words, 
the distinction between the normalized and the unnormalized equations is a genuine distinction.

First, a preliminary remark. Instead of assuming $f$ decreasing in the supplement to Theorem 3, it would suffice to assume $e^{-\kappa r} f(r)$ decreasing for a suitable positive constant $\kappa$. Namely, let $\kappa \neq \sigma$ and let

$$
P=(\sigma-\kappa) /(\sigma-\tau), \quad Q=(\kappa-\tau) /(\sigma-\tau)
$$

so that $P+Q=1$. Then a sufficient condition for $k$ is

$$
(\sigma Q+\tau P)^{2} \leqslant\left(\sigma^{2}\right)^{Q}\left(\tau^{2}\right)^{P} \quad(\kappa<\sigma)
$$

or the reversed inequality if $\kappa>\sigma$. The choice $\kappa=\sigma$ is permissible if $\sigma \leqslant \xi|\tau|$, where $\xi$ is the root of the transcendental equation $\xi \ln \xi=1+\xi$.

The proof of these remarks is left to the reader. Our object is to point out that any counterexample to Theorem 3 in the unnormalized case will require a rather irregular behavior both in $f$ and in the function $\lambda$.

We consider first the case $\beta=0$. By a change of scale it can be supposed that $2 \alpha=1$, and hence the desired condition for a radial solution $u(x)=\mu(r)$ is $\lambda \mu^{\prime \prime}-\mu^{\prime} \geqslant f$. This assumes equality in conditions (i) and (ii) of Theorem 3 , as is appropriate for construction of an example.

Let $w$ be a given positive continuous function which remains fixed throughout the discussion. We are going to find functions $f$ and $\lambda$ such that

$$
\int_{0}^{\infty} w(t) f(t) d t=\infty
$$

and nevertheless the solutions $u(x, R)$ of the above inequality do not satisfy $u(x, R) \leqslant 0$ for large $|x|$ and $R$. Hence, $u^{+}$does not have compact support. Condition (30) shows that no integral-divergence hypothesis bearing on $f, \alpha, \beta$ alone can suffice. For example, it would not be sufficient to have $f$ so large that $\int_{0}^{\infty} e^{-\delta t} f(t) d t=\infty$ for every $\delta$. This is seen by taking $w(t)=\exp \left(-t^{2}\right)$.

We choose a sequence $\left\{a_{n}\right\}$ which remains fixed throughout the discussion. For ease in following the proof one should consider that $a_{n}$ is sparse; say $a_{n}=$ $n$ ! However the only condition really needed is $a_{0}>0, a_{n+1}-a_{n} \geqslant 1$.

The function $f$ is to be positive near $a_{n}$ and 0 elsewhere, in such a way that the area of the spike near $a_{n}$ is $b_{n}$. Hence

$$
f(t) \doteq \sum_{n=1}^{\infty} b_{n} \delta\left(t-a_{n}\right)
$$

where $\delta$ is the Dirac distribution.

Next we take a large, smooth, increasing function $g(t)$ and define $1 / \lambda$ to agree with $g$ except in the immediate neighborhood of the points $a_{n}$, where $\lambda=$ 1. Then $f / \lambda=f$ and the desired inequality is 


$$
\mu^{\prime \prime}-g_{0} \mu^{\prime} \geqslant f
$$

with $g_{0}=g$ except in the immediate neighborhood of $a_{n}$, where $g_{0}=1$. We set

$$
G(t)=\int_{0}^{t} g_{0}(s) d s \doteq \int_{0}^{t} g(s) d s
$$

and note that the distinction between $g$ and $g_{0}$ is unimportant in (32), although it is important in (31).

Next pick $b_{n}$ so that

$$
b_{n} \geqslant 1, \sum_{n=1}^{\infty} w\left(a_{n}\right) b_{n}=\infty .
$$

The first condition is merely a convenience, but the second is needed to ensure (30). Finally, choose $g$ so large that

$$
g\left(a_{n-1}\right)>b_{n} 2^{n}, \quad n=1,2,3, \ldots .
$$

If the spikes around $a_{n}$ are narrow enough, it will be seen that $\mu$ gives a counterexample.

Indeed, the solution of the differential equation corresponding to (31) is

$$
\mu(r)=\int_{r}^{\rho} e^{G(t)} \int_{t}^{\rho} e^{-G(s)} f(s) d s d t
$$

where $\rho=R$ and $u(x, R)=\mu(r)$. (The condition $\mu^{\prime}(\rho)=0$ is irrelevant.) If $a_{m}$ is the first of the $a_{n}$ 's satisfying $a_{n} \geqslant t$ we have

$$
\int_{t}^{\rho} e^{-G(s)} f(s) d s=b_{m} e^{-G\left(a_{m}\right)}[1+E]
$$

where the error term $E$ satisfies

$$
E<b_{m+1} e^{-g\left(a_{m}\right)}+b_{m+2} e^{-g\left(a_{m+1}\right)}+\cdots \leqslant 1 .
$$

Here we have used $e^{-g}<1 / g$ and (33). If we estimate $\mu(r)$ in (34) by the above inequality on $\left(a_{m-1}, a_{m}\right)$ the result is

$$
\mu(r) \leqslant \sum_{m=1}^{\infty} \frac{2 b_{m}}{g\left(a_{m-1}\right)}<2 .
$$

Hence $\mu(r)$ remains bounded as $\rho \rightarrow \infty$ and the desired counterexample is obtained.

We now construct a similar example when $\beta \neq 0$. Here the family $u(x, R)$ $=\mu(x, \rho)$ is to satisfy $\lambda \mu^{\prime \prime}-2 \alpha \mu^{\prime}-\beta^{2} \mu \geqslant f$ where $\alpha>0$ and $\beta>0$ are given, but $\lambda$ with $0 \leqslant \lambda(x) \leqslant 1$ and $f$ satisfying (30) are to be found. To this end define

$$
-\tau=\left(\alpha^{2}+\beta^{2}\right)^{1 / 2}-\alpha, \quad \alpha_{0}=\alpha-\tau, f_{0}=f e^{-\tau t}, \quad w_{0}=w e^{\tau t} .
$$

The construction just described gives suitable functions $\mu_{0}, \lambda, f_{0}$ satisfying

$$
\lambda \mu_{0}^{\prime \prime}-2 \alpha_{0} \mu_{0}^{\prime} \geqslant f_{0}, \int_{0}^{\infty} w_{0}(t) f_{0}(t) d t=\infty .
$$


Then the family of functions $u(x, R)=\mu(r, \rho)=e^{\tau r} \mu_{0}(r, \rho)$ provides the desired example.

15. Proof of Theorem 4. Here the comparison equation is

$$
\mu^{\prime \prime}<f(\mu) g\left(\left|\mu^{\prime}\right|\right), \quad \mu^{\prime} \leqslant 0, \quad \mu^{\prime \prime} \geqslant 0,
$$

where the latter condition is needed to get the supplementary remark. Replacing $f$ by $f / 2$, we can get strict inequality in Lemma 1 from equality in (35); since $\left|\mu^{\prime}\right|>0$ for the function $\mu$ used here, the strict inequality is not needed at points where $\mu^{\prime}=0$.

Hence, we solve (35) with equality instead of inequality. The function $\xi=-\mu^{\prime}$ satisfies

$$
\int_{0}^{\xi} \frac{t d t}{g(t)}=\int_{0}^{\mu} f(t) d t
$$

Solving for $\xi$ gives, in the notation of Theorem $4,-d \mu / d r=G^{-1}[F(\mu)]$ and the desired condition $\mu(\rho)=0$ can be attained because the relevant integral converges near 0. Equation (36) shows that $\left|\mu^{\prime}\right|$ will remain small if $\int_{0}^{\infty} f(t) d t<\epsilon_{0}$ for $\epsilon_{0}$ sufficiently small, and this can always be achieved by use of a suitable minorant for $f$.

16. Conditional compactness. If the integral criterion in one of the above theorems fails there is a critical value $M_{c}$ such that every solution with $M<M_{c}$ has compact support, but the problem admits a counterexample with $M=M_{c}$. The problem is conditionally compact, and $M_{c}$ is the critical value.

In computing $M_{c}$ we ignore the restrictions associated with $\Omega(M, \epsilon)$. Thus the inequality is taken in the simple form

$$
a(x) u^{\prime \prime}-b(x) u^{\prime} \geqslant c\left(x, u, u^{\prime}\right)
$$

where $c\left(x, u, u^{\prime}\right)$ has the values $f(r), \beta^{2} u+f(r), f(u) g\left(\left|u^{\prime}\right|\right)$ in Theorems 2,3 or 4 , respectively. It is supposed that the hypotheses relevant to compactness holds for $|x| \geqslant r_{0}$; the region $|x| \leqslant r_{0}$ is taken care of by the basic hypothesis, as before.

Here are some critical values:

$$
\begin{array}{ll}
M_{c}=\int_{r_{0}}^{\infty} \frac{t^{2-N}-r_{0}^{2-N}}{2-N} t^{N-1} f(t) d t & (\text { Theorem 2, } N \neq 2), \\
M_{c}=\int_{r_{0}}^{\infty}\left[\log t-\log r_{0}\right] t f(t) d t & (\text { Theorem 2, } N=2), \\
M_{c}=\int_{r_{0}}^{\infty} \frac{e^{\tau\left(r_{0}-t\right)}-e^{\sigma\left(r_{0}-t\right)}}{\sigma-\tau} f(t) d t & (\text { Theorem } 3, \beta \neq 0), \\
M_{c}=\int_{r_{0}}^{\infty} \frac{e^{2 \alpha t}-e^{2 \alpha r_{0}}}{2 \alpha} e^{-2 \alpha t} f(t) d t & (\text { Theorem 3, } \beta=0) .
\end{array}
$$


With equal ease, one can get a relation between $M$ and the quantity $r_{0}+\rho$ which gives the radius of the support of $u^{+}$. For example under the hypothesis of Theorem 4 the relation is

$$
\rho=r_{0}+\int_{0}^{M} \frac{d s}{G^{-1}[F(s)]} .
$$

If the integral on the right converges as $M \rightarrow \infty$ the radius of the support of $u^{+}$ can be chosen independently of $M$. The corresponding phenomenon in Theorems 2 and 3 is not interesting because it requires a singularity in $f$. Here, however, the integral can converge even if $f$ and $g$ are regular; the singular behavior is generated by the nonlinearity in the term $f(u) g\left(\left|u^{\prime}\right|\right)$.

The above formula is sharp, in the sense that if $M$ is larger or $\rho$ smaller than allowed by the formula there exists a differential inequality and a corresponding solution providing a counterexample, much as in $\$ 14$. However the formula is not sharp as a function of $N$, because the inequality $N \geqslant 1$ was replaced by $N=$ 1 in the derivation. If $N>1$, the term $(N-1) / r$ in the comparison equation can be exploited, in general, so as to allow a larger $M$.

We give an example to show that this lack of sharpness is not always important. Let $f(t)=t^{\alpha}, 0<\alpha<1$, and let $g(s)=1$. Then the formula (37) gives

$$
\rho-r_{0}<\gamma M^{(1-\alpha) / 2}, \quad N \geqslant 1,
$$

where $\gamma=2 /(1-\alpha)$. On the other hand if $N$ is given in advance and $\gamma<\sqrt{2}$, the above estimate can fail, for some $M>0$, no matter how large $N$ may be. We omit the easy proof.

17. The main theorem. The foregoing boundary conditions exclude problems of Type II, in which $u_{\nu}$ alone is prescribed. Nor do they allow mixed conditions such as $p(x) u-q(x) u_{\nu} \leqslant 0(p \geqslant 0, q \geqslant 0, p+q=1)$. To generalize the boundary conditions let $\Gamma$ be divided into three parts, $\Gamma_{0}, \Gamma_{1}$ and $\Gamma_{2}$. We assume here that $\Gamma_{1}$ is compact, $\Gamma_{2}$ is bounded, and $\Gamma_{0}$ is the rest of $\Gamma$. Since $\Gamma_{2}$ will be primarily concerned with $u_{\nu}$, it requires some hypothesis of smoothness. We assume $\Gamma_{2}$ is regular in the following sense: there exists a function $\phi(x) \in C^{(2)}$ in a neighborhood of $\Gamma_{2}$ such that $\phi_{\nu}>0$ on $\Gamma_{2}$. This condition is discussed in [5]; suffice it to say here that $\Gamma_{2}$ can have any number of corners, provided the corners are not infinitely sharp. It is by no means required that $\Gamma_{2}$ admit an internally tangent paraboloid in the sense of Alexandrov, still less that it admit an internally tangent sphere in the sense of Hopf. The latter conditions do not even hold for a cube.

NEW BOUNDARY HYPOTHESIS. With $\Gamma=\Gamma_{0} \cup \Gamma_{1} \cup \Gamma_{2}$ as above, $u \leqslant 0$ on $\Gamma_{0}$ and, for some positive constants $M_{1}, M_{2}$, 


$$
x \in \Gamma_{1} \text { and } u>M_{1} \Rightarrow u_{\nu}>0, \quad x \in \Gamma_{2} \text { and } u>M_{2} \Rightarrow u_{\nu} \geqslant 0 \text {. }
$$

The foregoing proofs were simple because we had a rather strong hypothesis on the function $c$. The following allows a much broader class of functions $c$, at the cost of only a slight restriction on the matrix $a$ :

NEW INTERIOR HYPOTHESIS. There exists a positive constant $M_{0}$, independent of $R$, and a positive constant $\epsilon$, depending on $R$, such that the three conditions $x \in \Omega(R), u>M_{0},|p|<\epsilon$ together imply

$$
\begin{gathered}
\epsilon|a(x, u, p)|<1, \quad \epsilon|b(x, u, p)|<1, \quad \epsilon c(x, u, p) \geqslant-|p|, \\
p a(x, u, p) p \geqslant \epsilon|p|^{2}, \quad a(x, u, p) \geqslant 0 .
\end{gathered}
$$

The function $\beta(u)$ in Brezis' formulation has discontinuities at which the differential inequality may fail, and a term sgn $u$ occurs in the variational problems mentioned in the introduction. For such problems the inequality fails at the isolated zeros of $u$.

To deal with these matters, let $b_{i}$ be given numbers and let $B_{i}=\{x \mid u(x)=$ $b_{i}$ \}. We allow an exceptional set given by the countable union $B=\bigcup B_{i}$. Thus, instead of being a monotone graph $\beta(u)$ is allowed to be the interval $[-\infty, \infty]$ at each point of the countable set $\left\{b_{i}\right\}$. This case neither includes nor is included by that in [1].

NEW REgUlaRITY HYPOTHESIS. The functions $u(x, R)$ are of class $C^{0}$, $D^{1}$ and $D^{2}$ in the sets $\overline{\Omega(R)}, \Omega(R)$ and $\Omega(R)-B$, respectively. The differential inequality holds in the set $\Omega^{*}(\epsilon, R)=\Omega(\epsilon, R)-B$.

The following is the main theorem of this paper:

THEOREM 5. If the boundary, interior and regularity hypotheses are modified as described above, Theorems 1, 2, 3, 4 continue to hold.

18. Proof of the main theorem. In addition to the sets $B_{i}$ of the foregoing discussion, it is often possible to allow exceptional sets of the form $A_{i}=$ $\left\{x \mid a_{i}(x)=0\right\}$ where $a_{i}$ are given functions of class $D^{(2)}$ such that $\left|a_{i}^{\prime}(x)\right|>0$ in $\Omega(R)$. The differential inequality and the hypothesis $u \in D^{(2)}$ perhaps fail in the countable union $A=\bigcup A_{i}$.

This is not done here, because it would not be possible to ensure $p \| x$ at the maximum. Thus, introduction of the essential dimension would require some sort of continuity hypothesis, which we prefer to avoid. On the other hand for the special case $A_{i}=\left\{x|| x \mid=\alpha_{i}\right\}$ this objection does not apply, and such sets are allowed. They are useful whenever radial functions are pieced together, as in [1] and in the foregoing discussion. We take account of these sets in the proof of Theorem 5, though for simplicity they were omitted from the statement.

Lemma 4. Let $\Omega_{0}$ be a bounded open set and let $A$ and $B$ denote a count- 
able union of sets $\left\{x \mid a_{i}(x)=0\right.$ or $\left.u(x)=b_{i}\right\}$ as above. Let $u$ and $v$ be differentiable in $\Omega_{0}$, continuous in the closure, and let inf $\left|v^{\prime}(x)\right|>0$. Finally, suppose the maximum of $u-v$ over $\Omega_{0}$ does not occur on the boundary. Then there exists an arbitrarily small constant $\eta>0$ and an arbitrarily small function $\theta \in$ $C^{(2)}$, with $\theta^{\prime \prime}>0$, such that the maximum of $u-v+\eta u+\theta$ occurs at a point of $\Omega_{0}-A-B$. Furthermore, if the $a_{i}$ are radial, we can take $\theta$ to be radial.

To prove this let infl$\left|v^{\prime}\right|=2 \delta$ and let $B_{i}(\delta)=\left\{x \mid x \in \Omega_{0}, u(x)=b_{i}\right.$, $\left.\left|u^{\prime}(x)\right| \geqslant \delta\right\}$. Clearly it suffices to introduce a small perturbation which moves the maximum away from the union of the sets $A_{i}$ and $B_{i}(\delta)$. If we set $\psi_{2 n}=$ $a_{n}(x)$ and $\psi_{2 n+1}=u(x)$ this can be accomplished by a perturbation of form

$$
\psi(x)=\eta_{0}|x|^{2}+\sum_{n=1}^{\infty} \eta_{n} \psi_{n}(x)
$$

as in [4]. Lemma 4 follows at once.

LEMMA 5. Under the new interior, boundary and regularity hypotheses

$$
u(x, R) \leqslant M=\max \left(M_{0}, M_{1}, M_{2}\right)
$$

For proof assume $\max u(x, R)>M$. Then $\Gamma_{1}$ is excluded as a possible locus of the maximum. Furthermore the new interior hypothesis and Lemma 4 show that $u$ satisfies the strong maximum principle [3]. Hence the result follows by considering $u+\alpha e^{\beta \phi}$ in a shell-like region surrounding $\Gamma_{2}$, as in [3], [5], again using Lemma 4.

We now outline the proof of Theorem 5 . Let $\delta$ be a small positive constant and let $\mu_{1}(R)$ be a linear function whose graph contains the two points $(\delta, \rho)$ and $(R, 0)$. Also let $\mu_{0}(r)$ be chosen much as $\mu(r)$ was chosen in the proof of Theorems $1,2,3$, or 4 , except that the initial conditions $\mu(\rho)=\mu^{\prime}(\rho)=0$ formerly used are now replaced by $\mu_{0}(\rho)=\mu_{1}(\rho), \mu_{0}^{\prime}(\rho)=\mu_{1}^{\prime}(\rho)$. We define $\mu(r)=\mu_{0}(r)$ for $r_{0} \leqslant r \leqslant \rho$ and $\mu(r)=\mu_{1}(r)$ for $\rho \leqslant r \leqslant R$. This $\mu$ allows a larger $M$ than does the $\mu$ in the earlier proof, and it also satisfies $\left|\mu^{\prime}\right|>0, r_{0} \leqslant$ $r \leqslant R$. Hence we can use Lemma 4 with $v(x)=\mu(|x|)$. The small perturbation associated with Lemma 4 causes no trouble, because the functions $f$ and $g$ in our theorems are positive. Letting $\delta \rightarrow 0$ gives the desired conclusion.

The assumption that $f$ and $g$ are positive was introduced with a view to the argument above. For simpler results $f \geqslant 0$ and $g \geqslant 0$ would be sufficient.

19. Large comparison functions. Although the foregoing analysis allows boundary conditions of the form $u \leqslant M_{1}$ ( and indeed, this is the simplest case) a corresponding condition of form $u_{\nu} \geqslant-M_{2}$ is generally insufficient. Similarly, it is not enough just to have $c\left(x, u, u^{\prime}\right)$ bounded below in $|x| \leqslant r_{0}$, though this is a rather common situation in practice. 
Discussion of these matters is simplified if the boundary condition on $\Gamma_{1}$ is of Dirichlet type rather than mixed, and this is assumed here. Mixed conditions such as those above will require either that $\Gamma_{1} \cup \Gamma_{2}$ is regular or that the boundary operator $k\left(x, u, u_{v}\right)$ satisfies appropriate continuity conditions on $\Gamma_{1}$. The first case is included by letting $\Gamma_{1}$ be empty, so that $\Gamma_{2}$ stands for $\Gamma_{1} \cup \Gamma_{2}$. The second case is somewhat more complicated [5].

We also require the following

Topological ASSUMption. There exists a point $P$ in the complement of $\bar{\Omega}$ which can be joined to a point $Q$ in $\Omega$ by a continuous curve that does not meet $\Gamma_{2}$.

SuPPLEMENT. Suppose the hypotheses associated with Theorem 5 are modified as follows:

(i) $\Gamma_{1}$ is bounded, $\Gamma_{2}$ is regular and compact, and the associated boundary conditions are

$$
x \in \Gamma_{1} \Rightarrow u(x) \leqslant M_{1}, \quad x \in \Gamma_{2} \text { and } u(x)>M_{2} \Rightarrow u_{\nu}(x) \geqslant-M_{3} .
$$

(ii) The condition $\epsilon c(x, u, p) \geqslant-|p|$ in the interior hypothesis is replaced by $\epsilon c(x, u, p) \geqslant-1-|p|$, with $\epsilon=\epsilon(R)$ as before.

(iii) In all hypotheses pertaining to the region $|x| \leqslant r_{0}$ the side condition $|p|<\epsilon$ is dropped, and in all hypotheses pertaining to the region $|x| \geqslant r_{0}$ the side condition $u<M$ is dropped.

(iv) The topological assumption holds.

Then the conclusion still holds.

Here, as elsewhere, the $M_{k}$ are positive constants independent of $R$. In condition (iii) the value $r_{0}$ is fixed but sufficiently large. In particular, $|x|<$ $r_{0}$ contains $\Gamma_{1}, \Gamma_{2}$, the points $P, Q$ of the topological assumption, and their connecting curve. The value $r_{0}$ must also be allowed to satisfy $\epsilon r_{0}>1$, where $\epsilon$ is associated with the condition $\epsilon|x|>1$ in the set $\Omega(M, \epsilon)$. The essence of the matter is that an a priori bound $u<M$ is never available, and the condition $\left|u^{\prime}\right|<\epsilon$ is available only in the part of the analysis involving the radial comparison function $\mu(r), r_{0} \leqslant r \leqslant \rho<R$.

20. Outline of proof. Let $\theta$ be a function of class $C^{(2)}$ in $|x|<r_{0}+1$ which agrees with $\phi$ in a neighborhood of $\Gamma_{2}$ and agrees with $r_{0}-|x|$ in a neighborhood of $|x|=r_{0}$. Then move the singularities of $\theta$ in $\Omega$ to a small neighborhood of $P$, as is allowed by the topological assumption. Thus we get a function $\theta \in C^{(2)}$ in $\overline{\Omega\left(r_{0}\right)}$ satisfying

$$
\inf \theta_{\nu}>0 \text { on } \Gamma_{2}, \quad \text { inf }\left|\theta^{\prime}\right|>0 \text { in } \Omega\left(r_{0}\right) .
$$


Furthermore $\theta$ is radial near $|x|=r_{0}$ and $\partial \theta / \partial r<0,|x|=r_{0}$. We set

$$
v(x)=\gamma-\alpha e^{\beta \theta(x)}
$$

and try to choose the positive constants $\alpha, \beta$ and $\gamma$ in such a way that $u-v$ can attain a positive maximum in $|x| \leqslant r_{0}$ only on $|x|=r_{0}$, if at all. To this end, let $\alpha \geqslant 1$, and choose $\beta$ so that the maximum of $u-v$ cannot occur at an interior point of $\Omega\left(r_{0}\right)$ at which $u>M_{0}$. Next, increase $\alpha$, if necessary, so that $\alpha \beta e^{\beta \theta} \theta_{\nu}>M_{3}, x \in \Gamma_{2}$. This ensures that the maximum cannot occur at a point of $\Gamma_{2}$ where $u>M_{2}$. Finally, choose $\gamma$ so large that

$$
\gamma>M_{0}+M_{1}+M_{2}+\alpha e^{\beta \theta}, \quad x \in \Omega\left(r_{0}\right) \text {. }
$$

This ensures that $u$ is sufficiently large at a positive maximum of $u-v$ and completes the construction.

If $v=M$ on $|x|=r_{0}$ we find a suitable function $\mu(r)$ with $\mu\left(r_{0}\right)=M$ as in the proofs above. (Theorem 1 may require a linear function of small slope, before introduction of the main comparison function, because the value of $r_{0}$ must not be changed.) We now define $w(x)=v(x)$ for $|x| \leqslant r_{0}, w(x)=\mu(|x|)$ for $r_{0} \leqslant|x| \leqslant \rho$, and $w(x)=0$ for $\rho<|x|$. If the maximum of $u(x, R)-$ $w(x)$ is positive, its only possible location is on a point of $|x|=r_{0}$ interior to $\Omega$. But this contradicts Lemma 3. Hence, $u \leqslant w$, and the supplement follows.

Throughout this discussion we have tacitly assumed that $u \in D^{(2)}$. Under the new regularity assumption it is necessary to introduce a small perturbation in each stage of the argument, as in $\S 18$. The details are not difficult and are left to the reader.

21. Discussion of the supplement. Here we consider the question whether the topological assumption is really necessary for the supplement to the main theorem. As a matter of fact it is not, provided the comparison functions $\mu(r)$ have a large slope at $r_{0}$, and provided the condition $|p|<\epsilon$ is dropped in the definition of $\Omega(M, \epsilon)$. In this case we make $\theta$ behave like (const) $+|x|$ near $|x|=r_{0}$, instead of like (const) $-|x|$, so that the singularities can be moved to the region $|x|>r_{0}$. If $\left|\mu^{\prime}\left(r_{0}\right)\right|$ is sufficiently large, for the comparison function $\mu$ used beyond $r_{0}$, the desired conclusion still follows from Lemma 3. Conditions ensuring that $\left|\mu^{\prime}\left(r_{0}\right)\right| \rightarrow \infty$ with $M$ and $\rho$ are readily found. Indeed, $\left|\mu^{\prime}\left(r_{0}\right)\right| \rightarrow$ $\infty$ holds in Theorem 2 if $N>2$. It holds in Theorem 3 if $\beta>0$ or if $\beta=0$ and $\alpha<0$. It holds in Theorem 4 if $f$ and $g$ satisfy

$$
\int_{0}^{\infty} f(t) d t=\infty \text { or } \int_{0}^{\infty} \frac{t}{g(t)} d t<\int_{0}^{\infty} f(t) d t
$$

A version of Theorem 1 with $\left|\mu^{\prime}\left(r_{0}\right)\right| \rightarrow \infty$ is easily obtained by following the suggestions in $\S 9$, though we shall not do so. 
It is most interesting that, in the absence of the topological assumption, the supplement requires a large value of $\left|\mu^{\prime}\left(r_{0}\right)\right|$, whereas the original theorem required a large value of $\mu\left(r_{0}\right)$. Indeed, the main difficulty in the original proof was to show that $\left|\mu^{\prime}\right|$ was small.

We now show that this distinction between Theorem 5 and the supplement is a genuine distinction. If $\left|\mu^{\prime}\left(r_{0}\right)\right|$ remains bounded, and the topological assumption fails, then the supplement can fail even when the condition $\left|\mu\left(r_{0}\right)\right| \rightarrow \infty$ of Theorem 5 is fulfilled.

Let $\Omega$ be the region $|x|>1$ and let $N=1$; more specifically, let

$$
x a x=|x|^{2}, \quad a x^{\prime}-b x=1 \text {. }
$$

For example this holds for $a u^{\prime \prime}=\Delta u$ and $b x=n-1$, showing that the behavior illustrated here does not depend on a pathological choice of $a$ or $b$. We also take $c\left(x, u, u^{\prime}\right)=f(r)$ where $f(r)=r^{-3 / 2} / 2$ for $r \geqslant 2$, so that

$$
\int_{2}^{\infty} f(r) d r<\infty, \int_{2}^{\infty} r f(r) d r=\infty
$$

The first condition is needed to keep $\left|\mu^{\prime}(2)\right|$ bounded and the second is the integral criterion of Theorem 2 for $N=1$.

Let us now define

$$
\begin{array}{ll}
u(x, R)=\alpha-r\left(2^{1 / 2}-R^{-1 / 2}\right), & 1 \leqslant r \leqslant 2, \\
u(x, R)=(r-R) R^{-1 / 2}-2\left(r^{1 / 2}-R^{1 / 2}\right), & 2<r \leqslant R,
\end{array}
$$

where $\alpha$ is chosen so as to make the function continuous at $r=2$. Since the differential equation for a radial solution $\mu(r)$ is $\mu^{\prime \prime}(r)=f(r)$ in the case $N=1$, $c=f(r)$ being considered here, it is seen that $a u^{\prime \prime}-b u^{\prime}=f(r)$ where $f(r)=$ $r^{-3 / 2} / 2$ for $r>2$ and $f(r)=0$ for $r<2$. Also $u \in C^{(2)}$ except on $|x|=2$ where $u \in C^{(1)}$. Clearly $u(x, R)=0$, and

$$
u_{\nu}=-\left(2^{-1 / 2}-R^{-1 / 2}\right)>-2^{-1 / 2}, \quad|x|=1 \text {. }
$$

Nevertheless the support of $u^{+}$is not compact. This shows that we cannot allow a boundary condition such as $u_{\nu}>-1$ without the topological assumption, even if $c\left(x, u, u^{\prime}\right) \geqslant 0$ everywhere.

We could have defined $u(x, R)$ by a quadratic for $1 \leqslant|x| \leqslant 2$ in such a way as to make $u_{\nu}>0$ on $|x|=1$. Since the slope remains bounded on $|x|=2$ as $R \rightarrow \infty$, the second derivative would remain bounded. Thus we cannot allow a condition such as $c(x, u, p) \geqslant-1$ without the topological assumption, even if $u_{\nu}>0$ on the whole of $\Gamma_{2}$.

\section{REFERENCES}

1. Haim Brezis, Solutions with compact support of variational inequalities, Uspehi 
Mat. Nauk 29 (1974), 103-108 = Russian Math. Surveys 29 (1974), 103-108.

2. Norman Meyers and James Serrin, The exterior Dirichlet problem for second order elliptic partial differential equations, J. Math. Mech. 9 (1960), 513-538. MR 22 \#200.

3. John Piepenbrink and Ray Redheffer, Nonlinear partial differential inequalities, Arch. Rational Mech. Anal. 36 (1970), 89-121. MR 40 \#6033.

4. R. M. Redheffer and E. G. Straus, Degenerate elliptic equations, Pacific J. Math. 14 (1964), 265-268. MR 29 \#4979.

5. R. M. Redheffer, Elementary remarks on problems of mixed type, J. Math. and Phys. 43 (1964), 1-14. MR 29 \#2549.

DEPARTMENT OF MATHEMATICS, UNIVERSITY OF CALIFORNIA, LOS ANGELES, CALIFORNIA 90024

Current address: Mathematisches Institut I, Universität Karlsruhe (TH), P-7500, Karlsruhe, Germany 